\title{
ON A PROBLEM OF ONO AND QUADRATIC NON-RESIDUES
}

\section{MING-GUANG LEU}

\section{§. Introduction}

Let $k$ be a quadratic field, $\Delta_{k}$ the discriminant and $M_{k}$ the Minkowski constant:

$$
M_{k}= \begin{cases}\frac{1}{2} \sqrt{\Delta_{k}} & \text { if } k \text { is real } \\ \frac{2}{\pi} \sqrt{-\Delta_{k}} & \text { if } k \text { is imaginary. }\end{cases}
$$

Consider the finite set of prime numbers

$$
\Pi_{k}=\left\{p \text {, rational prime; } p \leq M_{k}\right\} \text {. }
$$

There are exactly 8 fields for which $\Pi_{k}=\emptyset$. They make up an exceptional family:

$$
E_{8}=\{k=\boldsymbol{Q}(\sqrt{m}) ; m=-1, \pm 2, \pm 3,5,-7,13\} .
$$

For any $k$, let $\chi_{k}$ denote the Kronecker character. The character splits $\Pi_{k}$ into 3 disjoint parts:

$$
\begin{aligned}
& \Pi_{k}^{0}=\left\{p \in \Pi_{k} ; \chi_{k}(p)=0\right\}, \\
& \Pi_{k}^{-}=\left\{p \in \Pi_{k} ; \chi_{k}(p)=-1\right\}, \\
& \Pi_{k}^{+}=\left\{p \in \Pi_{k} ; \chi_{k}(p)=+1\right\} .
\end{aligned}
$$

We remind the reader that for a positive prime integer $p$

$$
\chi_{k}(p)= \begin{cases}\left(\frac{\Delta_{k}}{p}\right) & \text { if } p \neq 2, p \nmid \Delta_{k}, \\ (-1)^{\left(\Delta_{k}^{2}-1\right) / 8} & \text { if } p=2,2 \nmid \Delta_{k}, \\ 0 & \text { if } p \mid \Delta_{k} .\end{cases}
$$

Consider, next, the 3 families of fields:

Received May 23, 1988. 


$$
\begin{aligned}
K^{0} & =\left\{k ; \Pi_{k}=\Pi_{k}^{0}\right\}, \\
K^{-} & =\left\{k ; \Pi_{k}=\Pi_{k}^{-}\right\}, \\
K^{+} & =\left\{k ; \Pi_{k}=\Pi_{k}^{+}\right\}
\end{aligned}
$$

Ono's problem in [4] is to determine explicitly the 3 families. Since $E_{8}$ is common to all 3 families, it is enough to determine $K^{0}-E_{8}$, $K^{-}-E_{8}, K^{+}-E_{8}$, respectively. In the first case, the equality $K^{0}-E_{8}$ $=\{k=\boldsymbol{Q}(\sqrt{m}) ; m=-5, \pm 6,7,15, \pm 30\}^{1)}$ is settled in [4]. In the second case, the equality $K^{-}-E_{8}=\{k=\boldsymbol{Q}(\sqrt{m}) ; m=-11,-19,-43,-67$, $-163,21,29,53,77,173,293,437\}$ is almost settled by H.M. Stark [6] and M.-G. Leu [3]. (For more details, see [4].)

In this paper, we shall consider the third case and prove that

$$
K^{+}-E_{8}=\{k=\boldsymbol{Q}(\sqrt{m}) ; m=-15,-23,-47,-71,-119,17,33,73,97\}
$$

which is the equality (5) in [4] hinted by the machine computations. Since $\chi_{k}(2)=0$ for $m \equiv 2,3(\bmod 4), \chi_{k}(2)=-1$ for $m \equiv 5(\bmod 8)$, and $\chi_{k}(2)=1$ for $m \equiv 1(\bmod 8)$, we have $m \equiv 1(\bmod 8)$ for $k=\boldsymbol{Q}(\sqrt{m})$ in $K^{+}-E_{8}$. So the problem to determine $K^{+}-E_{8}$ is reduced to prove that $M_{k}$ is larger than the least quadratic non-residue modulo $|m|$ for certain numbers $m$ of type $m \equiv 1(\bmod 8)$. For $k=\boldsymbol{Q}(\sqrt{m})$, we define the following 3 disjoint classes:

$$
\begin{aligned}
C & =\left\{n \in Z ; \chi_{k}(n)=0\right\}, \\
C_{1} & =\left\{n \in Z ; \chi_{k}(n)=-1\right\}, \\
C_{2} & =\left\{n \in Z ; \chi_{k}(n)=+1\right\} .
\end{aligned}
$$

In the sequel, $\left(\frac{n}{Q}\right)$ will denote the Jacobi symbol, where $Q$ denotes a positive odd integer and $n$ is an integer such that $(n, Q)=1$. Note that $\chi_{k}(n)=\left(\frac{n}{\left|\Delta_{k}\right|}\right)$ when $\Delta_{k} \equiv 1(\bmod 4)$ and $\left(n, \Delta_{k}\right)=1$. Furthermore, $m$ will denote a square-free integer $\equiv 1(\bmod 8),[x]$ the integral part of a positive real number $x, q$ the least positive integer belonging to $C_{1}$ and $p, p_{1}, p_{2}$ the positive prime numbers.

We shall divide our argument into two parts. In $\S 1$ we shall consider the case $m<0$ and in $\S 2$ the case $m>0$. Before the main argument, we prove the following lemma which enables us to consider

1) In [4] Ono included erroneously $m=10$ in the set $K^{0}-E_{8}$. This was pointed out by M. Ishibashi. 
only the cases where either $m$ is a prime number or a product of two prime numbers.

LEMma 1. If $k=\boldsymbol{Q}(\sqrt{m}) \in K^{+}-E_{8}$, then

(1) for $m>0$, either $m=p$ or $m=p_{1} p_{2}$, and

(2) for $m<0$, either $m=-p$ or $m=-p_{1} p_{2}$.

Proof. Suppose that $m>0$ and $m=p_{1} p_{2} p_{3} \cdots p_{n}, n \geq 3$, where $p_{i}$ is prime for $i=1,2, \cdots, n$. Without loss of generality, one can assume that $p_{1}=\min \left\{p_{1}, p_{2}, \cdots, p_{n}\right\}$. Then we have $p_{1}^{2}<p_{1} p_{2} \cdots p_{n} / 4$ which implies that $p_{1} \leq M_{k}$. Since $\chi_{k}\left(p_{1}\right)=0$, we have $\Pi_{k} \neq \Pi_{k}^{+}$and so $k=\boldsymbol{Q}(\sqrt{m})$ $\notin K^{+}-E_{8}$ which proves the assertion in the case (1). Similarly one proves the assertion in the case (2),

Q.E.D.

\section{§. The case $m<0$}

Case 1. $m=-p_{1} p_{2}, p_{1} p_{2} \equiv 7(\bmod 8)$.

Without loss of generality, we can assume that $p_{1}<p_{2}$. Since $p_{1}>$ $M_{k}=\frac{2}{\pi} \sqrt{p_{1} p_{2}}$ for $k=\boldsymbol{Q}\left(\sqrt{-p_{1} p_{2}}\right) \in K^{+}-E_{8}$, we have $p_{2}<\frac{\pi^{2}}{4} p_{1}$.

We first prove the following lemma.

Lemma 2. For $k=\boldsymbol{Q}\left(\sqrt{-p_{1} p_{2}}\right) \in K^{+}-E_{8}$, we have $q<\frac{p_{1} p_{2}}{8}$ if $p_{1} p_{2}$ $>3000$.

Proof. Suppose, on the contrary, that $q \geq \frac{p_{1} p_{2}}{8}$ for some $p_{1} p_{2}>3000$, since $p_{1}<p_{2}<3 p_{1}$, we have $23<p_{1}<q$. So $\left(\frac{23}{p_{1} p_{2}}\right)=1$ by the minimality of $q$. Since $\left[\frac{p_{1} p_{2}}{24}\right]=\frac{p_{1} p_{2}}{24}-s$ for some positive real number $s<1$, we have

$$
\begin{aligned}
\frac{7}{8} p_{1} p_{2}+1 & <\frac{22}{24} p_{1} p_{2}<\frac{22}{24} p_{1} p_{2}+\left(\frac{p_{1} p_{2}}{24}-23 s\right) \\
& =23\left(\frac{p_{1} p_{2}}{24}-s\right) \\
& =23\left[\frac{p_{1} p_{2}}{24}\right]<p_{1} p_{2} \quad \text { for } p_{1} p_{2}>3000
\end{aligned}
$$


So we have $23\left[\frac{p_{1} p_{2}}{24}\right]=p_{1} p_{2}-x$ for some integer $x, 1 \leq x<\frac{p_{1} p_{2}}{8}$. Since $\left[\frac{p_{1} p_{2}}{24}\right]=\frac{p_{1} p_{2}}{24}-s \neq a p_{1}$ or $b p_{2}$ for any integers $a, b$ (otherwise, we have

$$
24>24 s=\left\{\begin{array}{l}
p_{1}\left(p_{2}-24 a\right) \\
\text { or } \\
p_{2}\left(p_{1}-24 b\right)
\end{array}>24\right.
$$

a contradiction), we have $\left(x, p_{1} p_{2}\right)=1$. Furthermore, $\left[\frac{p_{1} p_{2}}{24}\right]<\frac{p_{1} p_{2}}{8} \leq$ q. Hence, we have

$$
\begin{aligned}
1 & =\left(\frac{23}{p_{1} p_{2}}\right)\left(\frac{\left[\frac{p_{1} p_{2}}{24}\right]}{p_{1} p_{2}}\right) \\
& =\left(\frac{23\left[\frac{p_{1} p_{2}}{24}\right]}{p_{1} p_{2}}\right) \\
& =\left(\frac{p_{1} p_{2}-x}{p_{1} p_{2}}\right) \\
& =\left(\frac{-x}{p_{1} p_{2}}\right) \\
& =\left(\frac{-1}{p_{1} p_{2}}\right)\left(\frac{x}{p_{1} p_{2}}\right) \\
& =-1, \text { a contradiction, }
\end{aligned}
$$

Now we can prove Theorem 1 after the model of the proof of the theorem in $[2]^{2)}$

Theorem 1. For $k=\boldsymbol{Q}\left(\sqrt{-p_{1} p_{2}}\right) \in K^{+}-E_{8}$, we have $p_{1} p_{2} \leq 360000$.

Proof. Since $\sqrt{2}\left(p_{1} p_{2}\right)^{2 / 5}+8\left(p_{1} p_{2}\right)^{1 / 5}+18<\frac{2}{\pi} \sqrt{p_{1} p_{2}}=M_{k}$ for $p_{1} p_{2}>$ 360000 , it suffices to prove that

$$
q<\sqrt{2}\left(p_{1} p_{2}\right)^{2 / 5}+8\left(p_{1} p_{2}\right)^{1 / 5}+18
$$

for $k=\boldsymbol{Q}\left(\sqrt{-p_{1} p_{2}}\right) \in K^{+}-E_{8}$ and $p_{1} p_{2}>360000$.

2) In p. 108 of [2], there seems to be a gap of arguments in the choice of $a$ and the choice of $\alpha$ there. So we make our argument slightly different from [2]. Our inequality (1.1) is weaker than that of Hudson and Williams in [2] (the inequality (2.1)), but our (1.1) is enough to prove our theorems. 
Assume, on the contrary, that

$$
q \geq \sqrt{2}\left(p_{1} p_{2}\right)^{2 / 5}+8\left(p_{1} p_{2}\right)^{1 / 5}+18, \quad \text { for some } p_{1} p_{2}>360000 \text {. }
$$

Since $p_{1} p_{2} \equiv 7(\bmod 8)$, we have $\left(\frac{8}{p_{1} p_{2}}\right)=1$ and $\left(\frac{-1}{p_{1} p_{2}}\right)=-1=\chi_{k}(-1)$.

By Lemma 2, we have $q<\frac{p_{1} p_{2}}{8}$ and so the integers

$$
p_{1} p_{2}-8(q-1), \quad p_{1} p_{2}-8(q-2), \cdots, p_{1} p_{2}-8
$$

are all positive and belong to $C$ or $C_{1}$.

Let $r$ be an odd positive integer of the form

$$
r=\left[\frac{1}{2}\left(p_{1} p_{2}\right)^{1 / 5}\right]+\alpha
$$

where $\alpha$ is a positive integer $\leq 4$ to be chosen later.

Since

$$
\frac{1}{2}\left(p_{1} p_{2}\right)^{1 / 5}<r \leq \frac{1}{2}\left(p_{1} p_{2}\right)^{1 / 5}+4
$$

and $p_{1}>M_{k}=\frac{2}{\pi} \sqrt{p_{1} p_{2}}$ as $k=\boldsymbol{Q}\left(\sqrt{-p_{1} p_{2}}\right) \in K^{+}-E_{8}$, we must have

$$
r<p_{1} \quad \text { and } \quad r \leq q-1
$$

Let $h$ be the unique integer satisfying

$$
8 h \equiv 8 q-p_{1} p_{2}(\bmod r), \quad 1 \leq h \leq r .
$$

By (1.7), we may define an integer $n$ by

$$
n=\frac{p_{1} p_{2}-8(q-h)}{r} .
$$

From (1.6) and (1.7), we have $1 \leq h \leq q-1$ and $1 \leq h<p_{1}$, and so the numerator in (1.8) is one of the integers in (1.3), and hence $n$ is positive.

Now, let $l=\left[2\left(p_{1} p_{2}\right)^{1 / 5}\right]+8$ so that

$$
2\left(p_{1} p_{2}\right)^{1 / 5}+7<l<2\left(p_{1} p_{2}\right)^{1 / 5}+8 \text {. }
$$

Further, put

$$
a=\left[n^{1 / 2}\right]+1 .
$$

Then $n^{1 / 2}<a \leq n^{1 / 2}+1$ so that $(a-1)^{2} \leq n<a^{2}$. Finally, choose $\alpha$ such that 


$$
\text { either } r \equiv 1(\bmod 8) \text { or } r \equiv 5(\bmod 8) \text {. }
$$

Case (I) $r \equiv 1(\bmod 8)$.

(a) $a \equiv 0$ or $3(\bmod 4)$.

As we verify it soon, we have

$$
(n+8 l-8) r \leq p_{1} p_{2}-8,
$$

and so the integers $n r,(n+8) r, \cdots,(n+8 l-8) r$ appear in the sequence (1.3) (c.f. (1.8)) and the $l$ integers

$$
n, n+8, \cdots, n+8 l-8,
$$

belong either to $C$ or to $C_{1}$ because $\left(\frac{r}{p_{1} p_{2}}\right)=1$. These integers are $\equiv 7$ (mod 8). Now, the condition (1.12) is satisfied because by (1.2), (1.5), (1.7), (1.8) and (1.9), we have

$$
\begin{aligned}
(n+8 l-8) r & <p_{1} p_{2}-8 q+8 r+8 r\left(2\left(p_{1} p_{2}\right)^{1 / 5}+8\right)-8 r \\
& <p_{1} p_{2}-8 q+8\left(\frac{1}{2}\left(p_{1} p_{2}\right)^{1 / 5}+4\right)\left(2\left(p_{1} p_{2}\right)^{1 / 5}+8\right) \\
& <p_{1} p_{2}-8 \sqrt{2}\left(p_{1} p_{2}\right)^{2 / 5}-64\left(p_{1} p_{2}\right)^{1 / 5}-144 \\
& \quad+8\left(p_{1} p_{2}\right)^{2 / 5}+96\left(p_{1} p_{2}\right)^{1 / 5}+256 \\
& =p_{1} p_{2}-8\left(p_{1} p_{2}\right)^{2 / 5}(\sqrt{2}-1)+32\left(p_{1} p_{2}\right)^{1 / 5}+112<p_{1} p_{2}-8 .
\end{aligned}
$$

If $a \equiv 0(\bmod 4)$, we consider the sequence of integers

$$
(a+1)(a-1),(a+3)(a-3), \cdots,(a+2 b-1)(a-2 b+1)
$$

where $b$ is the largest integer such that

$$
(a+2 b-1)(a-2 b+1)>(a-1)^{2} ;
$$

if $a \equiv 3(\bmod 4)$, we consider the sequence of integers

$$
(a+2) a,(a+4)(a-2), \cdots,(a+2 c)(a-2 c+2)
$$

where $c$ is the largest integer such that

$$
(a+2 c)(a-2 c+2)>(a-1)^{2} .
$$

Since the integers in $(1.14)$ and in $(1.16)$ are $\equiv 7(\bmod 8)$, we see that the integers in (1.13) are in the same residue class modulo 8 as those in (1.14) and as those in (1.16). 
Next, we have $(a-1)^{2} \leq n<\frac{p_{1} p_{2}}{r}<2\left(p_{1} p_{2}\right)^{4 / 5}$, so $a<\sqrt{2}\left(p_{1} p_{2}\right)^{2 / 5}+$ $1<p_{1}$. Then we have

$$
\begin{aligned}
a+2 b-1<a+\sqrt{2 a-1} & <\sqrt{2}\left(p_{1} p_{2}\right)^{2 / 5}+\sqrt{2 \sqrt{2}}\left(\left(p_{1} p_{2}\right)^{1 / 5}+1\right)+1 \\
& <\min \left(p_{1}, q\right),
\end{aligned}
$$

by (1.2) and by the inequalities, $p_{1} p_{2}>360000, p_{1}>\frac{2}{\pi} \sqrt{p_{1} p_{2}}$. Therefore the integers in (1.14) belong to $C_{2}$. Similarly the integers in (1.16) belong to $C_{2}$.

Thus, subdividing the integer interval

$$
\begin{cases}{\left[(a-1)^{2},(a-1)^{2}+1, \cdots, a^{2}-2, a^{2}-1\right]} & \text { if } a \equiv 0(\bmod 4), \\ {\left[(a-1)^{2},(a-1)^{2}+1, \cdots, a^{2}+2 a-1, a^{2}+2 a\right]} & \text { if } a \equiv 3(\bmod 4)\end{cases}
$$

by the integers in (1.14) and (1.16), respectively, we see, by (1.13), that $8 l-8$ is less than the maximum difference between integers in the subdivided interval. This gives the required contradiction; we just give the details for $a \equiv 3(\bmod 4)$. In this case, the difference between integers in (1.16) in the subdivided interval of $\left[(a-1)^{2},(a-1)^{2}+1, \cdots, a^{2}+2 a\right.$ $\left.-1, a^{2}+2 a\right]$ is at most

$$
\begin{aligned}
(a+2 c)(a-2 c+2)-(a+2 c+2)(a-2 c) & =8 c \\
& <4+8 a^{1 / 2} \\
& <4+8 \sqrt[4]{2}\left(\left(p_{1} p_{2}\right)^{1 / 5}+1\right) \\
& <16\left(p_{1} p_{2}\right)^{1 / 5}+20 \\
& <8 l-8 .
\end{aligned}
$$

(b) $a \equiv 1$ or $2(\bmod 4)$.

If $a \equiv 2(\bmod 4)$, we consider the sequence of integers

$(1.14)^{\prime} \quad(a+3)(a+1),(a+5)(a-1), \cdots,(a+2 b+1)(a-2 b+3)$,

where $b$ is the largest integer such that

$$
(a+2 b-1)(a-2 b+1)>(a-1)^{2} ;
$$

if $a \equiv 1(\bmod 4)$, we consider the sequence of integers

$$
(a+6) a,(a+8)(a-2), \cdots,(a+2 c)(a-2 c+6)
$$

where $c$ is the largest integer such that 


$$
(a+2 c)(a-2 c+2)>(a-1)^{2} .
$$

By a similar argument as in (a), we also get a contradiction.

Similarly one wlll get a contradiction for Case (II) where $r \equiv 5(\bmod 8)$,

Q.E.D.

Case 2. $m=-p, p \equiv 7(\bmod 8)$.

By almost the same argument as in the proof of Theorem 1, we have the following theorem.

TheOREm $1^{\prime}$. For $k=\boldsymbol{Q}(\sqrt{-p}) \in K^{+}-E_{8}$, we have $p \leq 360000$.

According to Case 1 and Case 2, for $m<0$ and $k=\boldsymbol{Q}(\sqrt{m}) \in K^{+}-E_{8}$, $-m$ must be $\leq 360000$. By the help of a computer in our department, we obtain that $m=-15,-23,-47,-71,-119$. (See table).

\section{§. The case $m>0$}

Case $1 . \quad m=p, p \equiv 1(\bmod 8)$.

By applying a theorem of L. Rédei [5], we shall prove Proposition 1 below which will provide $\frac{\sqrt{p}}{2}$ as an upper bound for the least quadraticnonresidue of a prime $p \equiv 1(\bmod 8)$ for $p>97$.

Theorem (L. Rédei [5]). For $4 \mid p-1$, the density $\delta_{2}$ of the quadratic residues, and also the density $\delta_{1}$ of the non-residues $(\bmod p)$ in the interval $[1, \sqrt{p}]$ is grater than $\frac{1}{4+2 \sqrt{2}}$ and less than $1-\frac{1}{4+2 \sqrt{2}}$.

Proposition 1. For $p \equiv 1(\bmod 8)$ and $p>240000$ then $q<M_{k}=\frac{\sqrt{p}}{2}$.

Proof. Suppose, on the contrary, that there exists a prime $p_{0} \equiv 1$ $(\bmod 8), p_{0}>240000$ such that $q>\frac{\sqrt{p_{0}}}{2}$.

Let $x=\left[\sqrt{p_{0}}\right]$, the integral part of $\sqrt{p_{0}}$. Then one observes the following four cases.

(i) There are at least $\frac{x-a}{2}$ integers $<\frac{\sqrt{p_{0}}}{2}$ which are quadratic residues $\left(\bmod p_{0}\right)$, where $a$ is an integer, $0 \leq a \leq 1$, such that $\frac{x-a}{2}$ is an integer.

(ii) There are at least $\left(x-\frac{x+a^{\prime}}{2}\right) / 2$ even integers in the inter- 
$\operatorname{val}\left(\left[\frac{\sqrt{p_{0}}}{2}\right],\left[\sqrt{p_{0}}\right]\right)$, which are quadratic residues $\left(\bmod p_{0}\right)$, where $a^{\prime}$ is an integer, $0 \leq a^{\prime} \leq 3$, such that $x-\frac{x+a^{\prime}}{2}$ is an even integer. (Note that for an even integer $2 b<\sqrt{p_{0}}$, we have $b<\frac{1}{2} \sqrt{p_{0}}$ and so $b$ is a quadratic residue.)

(iii) There are at least $\left(\frac{x-b}{3}-\frac{x+b^{\prime}}{6}\right) / 2$ odd integers with 3 as a factor in the interval $\left(\left[\frac{\sqrt{p_{0}}}{2}\right],\left[\sqrt{p_{0}}\right]\right)$, which are quadratic residues $\left(\bmod p_{0}\right)$, where $b, b^{\prime}$ are integers, $0 \leq b, b^{\prime} \leq 5$, such that $\frac{x-b}{3}, \frac{x+b^{\prime}}{6}$ and $\left(\frac{x-b}{3}-\frac{x+b^{\prime}}{6}\right) / 2$ are integers.

(iv) There are at least $\frac{1}{3}\left\{\left(\frac{x-c}{5}-\frac{x+c^{\prime}}{10}\right)-d\right\}$ odd integers relatively prime to 3 with 5 as a prime factor in the interval $\left(\left[\frac{\sqrt{p_{0}}}{2}\right],\left[\sqrt{p_{0}}\right]\right)$, which are quadratic residues $\left(\bmod p_{0}\right)$, where $c, c^{\prime}$ and $d$ are integers, $0 \leq c, c^{\prime} \leq 9,0 \leq d \leq 2$, such that

$$
\frac{x-c}{5}, \frac{x+c^{\prime}}{10} \text { and } \frac{1}{3}\left\{\left(\frac{x-c}{5}-\frac{x+c^{\prime}}{10}\right)-d\right\}
$$

are integers.

From (i), (ii), (iii) and (iv), we see that there are at least

$$
\begin{aligned}
N=\frac{x-a}{2} & +\left(x-\frac{x+a^{\prime}}{2}\right) / 2+\left(\frac{x-b}{3}-\frac{x+b^{\prime}}{6}\right) / 2 \\
& +\frac{1}{3}\left\{\left(\frac{x-c}{5}-\frac{x+c^{\prime}}{10}\right)-d\right\}
\end{aligned}
$$

distinct integers in the interval $\left[1, \sqrt{p_{0}}\right]$, which are quadratic residues $\left(\bmod p_{0}\right)$. We have

$$
\begin{aligned}
N \geq & \frac{x-1}{2}+\left(x-\frac{x+3}{2}\right) / 2+\left(\frac{x-5}{3}-\frac{x+5}{6}\right) / 2 \\
& +\frac{1}{3}\left\{\left(\frac{x-9}{5}-\frac{x+9}{10}\right)-2\right\} \\
& =\frac{52 x-244}{60} .
\end{aligned}
$$


So the density $\delta_{2}$ of quadratic residues in $\left[1, \sqrt{p_{0}}\right]>\frac{N}{x} \geq \frac{1}{60}\left(52-\frac{244}{x}\right)$. Since $\frac{1}{4+2 \sqrt{2}}>\frac{1}{7}$, we have

$$
1=\delta_{1}+\delta_{2}>\frac{1}{7}+\frac{52-\frac{244}{x}}{60}=\frac{424-\frac{1708}{x}}{420}>1 \quad \text { for } p_{0}>240000
$$

a contradiction,

Q.E.D.

Case 2. $m=p_{1} p_{2}, p_{1} p_{2} \equiv 1(\bmod 8)$.

Without loss of generality, one can assume that $p_{1}<p_{2}$. Since $p_{1}>$ $M_{k}=\frac{\sqrt{p_{1} p_{2}}}{2}$ for $k=\boldsymbol{Q}\left(\sqrt{p_{1} p_{2}}\right) \in K^{+}-E_{8}$, we have $p_{2}<4 p_{1}$.

For $p_{1} p_{2} \equiv 1(\bmod 8), p_{1}<p_{2}<4 p_{1}$ and $p_{1} p_{2}>300$, by a theorem of Thue [1], the congruence $x \equiv n y\left(\bmod p_{1} p_{2}\right)$ has non-trivial solutions $x, y$ for which $|x| \leq \sqrt{p_{1} p_{2}}$ and $|y| \leq \sqrt{p_{1} p_{2}}$. We can choose a positive integer $n$ such that $n<p_{1} p_{2},\left(n, p_{1} p_{2}\right)=1, n \neq \equiv+1\left(\bmod p_{2}\right)$ and $\left(\frac{n}{p_{1} p_{2}}\right)=-1$. By the choice of $n$, we see that one of the numbers $x$ and $y$, say $x$, must belong to $C_{1}$. (Note that $p_{1}<\sqrt{p_{1} p_{2}}<2 p_{1}$, so neither $|x|$ nor $|y|$ equal to $p_{1}$, because otherwise $n \equiv+1$ or $-1\left(\bmod p_{2}\right)$, which contradicts the choice of $n$.) Since $\left(\frac{x}{p_{1} p_{2}}\right)=-1$, we have $\left(\frac{-x}{p_{1} p_{2}}\right)=-1$. So there exists a positive integer $x<\sqrt{p_{1} p_{2}}$ such that $\left(\frac{x}{p_{1} p_{2}}\right)=-1$. Denote by $v_{i}$ the number of elements in $C_{i}, i=1,2$, which lie in the interval $\left[1, \sqrt{p_{1} p_{2}}\right]$. Furthermore, since $\left(\frac{2}{p_{1} p_{2}}\right)=1$, we see that $v_{i} \neq 0, i=1,2$, for $k=$ $\boldsymbol{Q}\left(\sqrt{p_{1} p_{2}}\right) \in K^{+}-E_{8}$ and $p_{1} p_{2}>300$. We have $v_{1}+v_{2}=\left[\sqrt{p_{1} p_{2}}\right]-1$ because $p_{1}<\sqrt{p_{1} p_{2}}$. Denote by $\delta_{i}=\frac{v_{i}}{\left[\sqrt{p_{1} p_{2}}\right]-1}$ the density of the class $C_{i}$ in the interval $\left[1, \sqrt{p_{1} p_{2}}\right]$, for $i=1,2$. Now we are ready to prove Theorem 2 which is similar to a theorem of Rédei [5].

THEOREM 2. For $\left.k=\boldsymbol{Q} \sqrt{p_{1} p_{2}}\right)$, if $p_{1} p_{2} \equiv 1(\bmod 8), p_{1}<p_{2}<4 p_{1}$ and $p_{1}>265$, then we have $\frac{1}{7}<\delta_{1}, \delta_{2}<1-\frac{1}{7}$.

Proof. Since $\left(p_{1}-1\right)\left(p_{2}-1\right) / 2$ is the number of incongruent elements $\left(\bmod p_{1} p_{2}\right)$ in $C_{d}, d=1,2$, for $\alpha \in C_{1}$ with $\alpha \neq \equiv \pm 1\left(\bmod p_{2}\right)$, there exist $x$, 
$y, x \in C_{i}, y \in C_{j}, i \neq j, 1 \leq x, y<\sqrt{p_{1} p_{2}}$, such that $\alpha \equiv \frac{y}{x}$ or $-\frac{y}{x}(\bmod$ $p_{1} p_{2}$ ). From this, we have

$$
2\left(v_{1} v_{2}+v_{2} v_{1}\right) \geq \frac{\left(p_{1}-1\right)\left(p_{2}-1\right)}{2}-2 p_{1}
$$

where $2 p_{1}$ is the number of elements in the set

$$
\left\{n \in N ; n<p_{1} p_{2}, n \equiv+1 \text { or }-1\left(\bmod p_{2}\right)\right\} \text {. }
$$

Then since $v_{1}+v_{2}=\left[\sqrt{p_{1} p_{2}}\right]-1$, one has, by $(2.1)$,

$$
\begin{aligned}
\delta_{1} \delta_{2}+\delta_{2} \delta_{1} \geq x \quad & \left(x=\frac{\left(p_{1}-1\right)\left(p_{2}-1\right)-4 p_{1}}{4\left(\left[\sqrt{p_{1} p_{2}}\right]-1\right)^{2}}\right) \\
& \delta_{1}+\delta_{2}=1 .
\end{aligned}
$$

Consider the equations:

$$
\begin{gathered}
2 u v=x \\
u+v=1
\end{gathered}
$$

One solution for (2.4), (2.5) is

$$
u=\frac{1+\sqrt{1-2 x}}{2}, \quad v=\frac{1-\sqrt{1-2 x}}{2}
$$

by which the square root may be chosen positive because $2 x<1$ for $p_{1}>265$.

We set

$$
\delta_{1}=\frac{v_{1}}{\left[\sqrt{p_{1} p_{2}}\right]-1}=u+\alpha_{1}, \quad \delta_{2}=\frac{v_{2}}{\left[\sqrt{p_{1} p_{2}}\right]-1}=v+\alpha_{2}
$$

where $\alpha_{1}, \alpha_{2}$ are real numbers. By (2.3), (2.5) we have

$$
\alpha_{1}+\alpha_{2}=0 \text {. }
$$

Furthermore, it follows from (2.2) that

$$
2 \delta_{1} \delta_{2} \geq x
$$

i.e., by (2.3), $\delta_{1}^{2}+\delta_{2}^{2} \leq 1-x$.

By (2.7) we have

$$
u^{2}+v^{2}+2 u \alpha_{1}+2 v \alpha_{2}+\left(\alpha_{1}^{2}+\alpha_{2}^{2}\right) \leq 1-x .
$$


Since $u^{2}+v^{2}=1-x$ by (2.6) and $\alpha_{1}^{2}+\alpha_{2}^{2} \geq 0$, we have $2 u \alpha_{1}+2 v \alpha_{2} \leq 0$. By (2.8), we also have $2 u \alpha_{1}-2 v \alpha_{1} \leq 0$. On the other hand it follows from (2.6) that $u-v>0$, and so $\alpha_{1} \leq 0$, i.e., by (2.7), $\delta_{1} \leq u$. Because the conditions (2.2), (2.3) are symmetric in $\delta_{1}, \delta_{2}$, one has $\delta_{i} \leq u, i=1,2$. Furthermore, we have $\left(\left[\sqrt{p_{1} p_{2}}\right]-1\right)^{2} \leq \frac{65}{64}\left[\left(p_{1}-1\right)\left(p_{2}-1\right)-4 p_{1}\right]$ because $p_{1}>265$ and, by (2.2), we have $2 x \geq \frac{32}{65}$. Therefore, by (2.6), we have

$$
\begin{aligned}
\delta_{i} \leq u & \leq\left(1+\sqrt{\frac{33}{65}}\right) / 2 \\
& \approx 0.8562 \ldots \\
& \leq 1-\frac{1}{7} \approx 0.8571 \cdots
\end{aligned}
$$

where $i=1,2$,

Q.E.D.

By the similar argument as the proof of Proposition 1, we have the following proposition:

Proposition 2. Assume that $k=\boldsymbol{Q}\left(\sqrt{p_{1} p_{2}}\right), p_{1} p_{2} \equiv 1(\bmod 8), p_{1}<p_{2}$ $<4 p_{1}, p_{1}>265$ and $p_{1} p_{2}>240000$. Then $q \leq M_{k}=\frac{\sqrt{p_{1} p_{2}}}{2}$.

According to Proposition 1 and Proposition 2, we see that for $m>0$ and $k=\boldsymbol{Q}(\sqrt{ } \bar{m}) \in K^{+}-E_{8}, m$ must be less than 290000. With the help of computer, we obtain that $m=17,33,73,97$. (See table.)

Combining the results in $\S 1$ and $\S 2$, we have proved that

$$
K^{+}-E_{8}=\{k=\boldsymbol{Q}(\sqrt{m}) ; m=-15,-23,-47,-71,-119,17,33,73,97\} .
$$


$\mathrm{Table}^{3)}$

\begin{tabular}{|c|c|c|c|c|c|c|c|}
\hline \multicolumn{4}{|c|}{$m>0$} & \multicolumn{4}{|c|}{$m<0$} \\
\hline$m=0$ & $q$ & $m=p_{1} p_{2}$ & $q$ & $-m=p$ & $q$ & $-m=p_{1} p_{2}$ & $q$ \\
\hline 17 & - & 33 & - & 23 & - & 15 & - \\
\hline 41 & 3 & 65 & 3 & 31 & 3 & 55 & 3 \\
\hline 73 & - & 161 & 3 & 47 & - & 119 & - \\
\hline 89 & 3 & 209 & 3 & 71 & - & 143 & 5 \\
\hline 97 & - & 377 & 3 & 79 & 3 & 247 & 3 \\
\hline 113 & 3 & 473 & 3 & 103 & 3 & 391 & 3 \\
\hline 137 & 3 & 481 & 7 & 127 & 3 & 527 & 5 \\
\hline 193 & 5 & 697 & 5 & 151 & 3 & 551 & 11 \\
\hline 233 & 3 & 713 & 3 & 167 & 5 & 703 & 3 \\
\hline 241 & 7 & 817 & 5 & 191 & 7 & 943 & 3 \\
\hline 257 & 3 & 1073 & 3 & 199 & 3 & 1247 & 5 \\
\hline 281 & 3 & 1081 & 7 & 223 & 3 & 1271 & 7 \\
\hline & $\vdots$ & & $\vdots$ & $\vdots$ & $\vdots$ & : & $\vdots$ \\
\hline : & : & & : & : & : & & : \\
\hline 239641 & 7 & 239969 & 3 & 359311 & 3 & 356047 & 3 \\
\hline 239689 & 11 & 240809 & 3 & 359327 & 5 & 356359 & 3 \\
\hline 239713 & 5 & 241697 & 3 & 359407 & 3 & 356519 & 7 \\
\hline 239737 & 5 & 243721 & 19 & 359479 & 3 & 356639 & 19 \\
\hline 239753 & 3 & 244921 & 7 & 359599 & 3 & 357191 & 7 \\
\hline 239849 & 3 & 251089 & 7 & 359663 & 5 & 357407 & 5 \\
\hline 239857 & 5 & 254321 & 3 & 359719 & 3 & 358151 & 17 \\
\hline 239873 & 3 & 258529 & 7 & 359767 & 3 & 358871 & 7 \\
\hline 239929 & 11 & 259313 & 3 & 359783 & 5 & 359039 & 7 \\
\hline 239977 & 5 & 260633 & 3 & 359911 & 3 & 359831 & 19 \\
\hline 240017 & 3 & 271153 & 5 & 360007 & 3 & 359903 & 5 \\
\hline 240041 & 3 & 273257 & 3 & 360023 & 5 & 359999 & 17 \\
\hline
\end{tabular}

3) In the column " $q$ " of the table, the smallest odd prime $q \leq M_{k}$ such that $\chi_{k}(q)=-1$ is given. Since the complete table would occupy at least 20 pages long, we only show the beginning and the end of the original table. 


\section{REFERENCES}

[1] A. Brauer, Combinatorial Methods in the Distribution of $k$-th Power Residues, Combinatorial Mathematics and its Applications, Chapel Hill 1967, 14-37.

[2 ] R. H. Hudson and K. S. Williams, On the Least Quadratic Nonresidue of a prime $p \equiv 3(\bmod 4)$, J. Reine Angew. Math., 318 (1980), 106-109.

[ 3 ] M.-G. Leu, On a Conjecture of Ono on Real Quadratic Fields, Proc. Japan Acad., 63A (1987), 323-326.

[4] T. Ono, A Problem on Quadratic Fields, Proc. Japan Acad., 64A (1988), 78-79.

[ 5 ] L. Rédei, Über die Anzahl der Potenzreste mod $p$ im Intervall, $1, \sqrt{ } \frac{(}{p}$ Nieuw Arch. Wiskunde, (2) 23 (1950), 150-162.

[6] H. M. Stark, A complete determination of the complex quadratic fields of classnumber one, Michigan Math. J., 14 (1967), 1-27.

Department of Mathematics

The Johns Hopkins University

Baltimore, $M D$ 21218

U.S.A.

Permanent address :

Department of Mathematics

National Central University

Chung-Li, Taiwan 32054

R. O.C. 